\title{
Iterative solution of split equilibrium and fixed point prob- lems in real Hilbert spaces
}

\author{
J. N. Ezeora*, P. C. Jackreece \\ Department of Mathematics and Statistics, University of Port Harcpourt, Nigeria.
}

\begin{abstract}
In this article, we introduce a hybrid iteration involving inertial-term for split equilibrium problem and fixed point for a finite family of asymptotically strictly pseudocontractive mappings. We prove that the sequence converges strongly to a solution of split equilibrium problem and a common fixed point of a finite family of asymptotically strictly pseudocontractive mappings. The results proved extend and improve recent results of Chang et al. [S. S. Chang, H. W. J. Lee, C. K. Chan, L. Wang, L. J. Qin, Appl. Math. Comput., 219 (2013), 10416-10424], Dewangan et al. [R. Dewangan, B. S. Thakur, M. Postolache, J. Inequal. Appl., 2014 (2014), 11 pages], and many others.
\end{abstract}

Keywords: Total asymptotically strict pseudocontractive mapping, split equilibrium problem, fixed point problem, inertial-step, bounded linear operator.

2020 MSC: 47H09, 47H10, 49M05, 54H25.

(C)2021 All rights reserved.

\section{Introduction}

Fixed point theory of nonexpansive mappings has valuable applications in different fields such as; convex feasibility problems, convex optimization problems, approximation theory, game theory, signal and image processing, partial differential equations and so on (see for example $[16,33]$ and the references therein). Existence of solutions associated with the above-mentioned problems depends on the existence of fixed points of certain noinlinear mappings.

In 1994, Censor and Elfving [11] introduced in finite dimensional real Hilbert spaces, the split feasibility problems (SFP) for modeling inverse problems which arise from phase retrievals and in medical image reconstruction. It is now known that SFP can be applied in many disciplines such as; image restoration, computer tomograph and radiation therapy treatment planning (see $[8,9,12,13])$. Consequently, the study of SFP has received the attention of many researchers (see $[4,27,34,36]$ and the references therein).

Let $\mathrm{H}_{1}$ and $\mathrm{H}_{2}$ be two real Hilbert spaces and $\mathrm{C}$ and $\mathrm{Q}$ be nonempty closed convex subsets of $\mathrm{H}_{1}$ and $\mathrm{H}_{2}$, respectively. The SFP is formulated as follows; find a point $\mathrm{q} \in \mathrm{H}_{1}$ such that

$$
q \in C \text { and } A q \in Q \text {, }
$$

\footnotetext{
${ }^{*}$ Corresponding author

Email addresses: jeremiah.ezeora@uniport.edu.ng (J. N. Ezeora), prebo.jackreece@uniport.edu.ng (P. C. Jackreece)

doi: $10.22436 /$ jnsa.014.05.06
}

Received: 2021-01-22 Revised: 2021-02-27 Accepted: 2021-03-18 
where $A: H_{1} \rightarrow H_{2}$ is a bounded linear operator. If (1.1) has solution, it can be shown that $x \in C$ solves (1.1) if and only if it solves the following fixed point equation:

$$
x=P_{C}\left(\left(I-\gamma A^{*}\left(I-P_{Q}\right)\right) A\right) x,
$$

where $P_{C}$ and $P_{Q}$ are the metric projections onto $C$ and $Q$, respectively, $\gamma$ is a positive constant and $A^{*}$ denotes the adjoint of $A$. Under appropriate conditions on $\gamma$, it can be shown that the operator $\mathrm{P}_{\mathrm{C}}((\mathrm{I}-$ $\gamma A^{*}\left(I-P_{Q}\right) A$ associated with (1.2) is nonexpansive.

For an equilibrium bifunction $\mathrm{f}: \mathrm{C} \times \mathrm{C} \rightarrow \mathbb{R}$, the equilibrium problem is defined as; find $\mathrm{x} \in \mathrm{C}$ such that $f(x, y) \geqslant 0, \forall y \in C$. We remark that iterative solution of equilibrium problems and fixed point problems has been studied extensively in the last three decades or so (see $[2,6,9,17,19,28,30,32])$.

For two equilibrium bifunctions $f$ and $g$ satisfying certain conditions (see Section 2 below), the split equilibrium problem (SEP) entails finding a solution of an equilibrium problem, $x^{*} \in \operatorname{EP}(f)$ such that the image of $x^{*}$ under a bounded linear map, $A x^{*}$ is a solution of another equilibrium problem $\operatorname{EP}(\mathrm{g})$. This problem has been studied and successfully employed as a model in intensity-modulated radiation therapy treatment planning, see for instance, $[4,10,23]$ and the references contained therein.

In 1967, Browder and Petryshyn [7] introduced the class of strict pseudo contractive maps as a generalization of the class of nonexpansive mappings. Other generalizations of nonexpansive mappings have also been introduced and studied in the literature (see Alber et al. [1] and Yang et al. [35]).

In [14], the authors studied SFP involving quasi-nonexpansive mapping and total asymptotically strict pseudo-contractive mapping. Other authors have also studied the same problem using different assumptions (see $[20,25,26,31]$ and the references therein). Unfortunately, the stepsize in these existing results often depend on the norm of the associated bounded linear operator or on the spectral radius of $A^{*} A$. This makes those results very difficult to use in any possible application.

Inertial-type algorithms are based on the heavy ball methods of the two-order time dynamical system. Polyak [29] first proposed an inertial extrapolation as a speed up process to solve the smooth convex minimization problem. The inertial algorithm is a two-step iterative method in which the next iteration is obtained by the previous two iterates, which improve the convergence rate of the iterative sequence (see $[5,21,29])$.

Inspired by the ongoing research explored above, it is our purpose in this article to design an inertialtype iterative algorithm in which the stepsize does not need prior knowledge of the norm of the associated bounded linear operator and prove strong convergence of the sequence for SEP and fixed point of total asymptotically strict pseudo-contractive mapping in real Hilbert spaces.

The rest of the paper is arranged as follows. In Section 2, we provide some definitions and preliminary results which will be needed throughout the paper. Sections 3 is devoted to the main result, while in Section 4, we apply the result of Section 3 to study some related problems.

\section{Preliminaries}

This section is devoted to recalling some fundamental definitions, properties and notations concerned with the split equilibrium problem and fixed point problem in real Hilbert spaces. Throughout the paper, $x_{k} \rightarrow x$ (resp. $\left.x_{k} \rightarrow x\right)$ denotes strong convergence (resp. weak convergence) of a sequence $\left\{x_{k}\right\}$. Except otherwise stated, $\mathrm{H}$ stands for a real Hilbert space and $\mathrm{C} \subseteq \mathrm{H}$ denotes a closed convex subset of $\mathrm{H}$.

Let $\mathrm{C}$ be a nonempty subset of a real Hilbert space $\mathrm{H}$ and let $\mathrm{T}: \mathrm{C} \rightarrow \mathrm{C}$ be a mapping. We denote by $F(T)$ the set of fixed points of the mapping $T$. That is, $F(T)=\{x \in C: T(x)=x\}$.

Definition 2.1. The mapping $T$ is said to be:

(i) nonexpansive if

$$
\|T x-T y\| \leqslant\|x-y\|, \forall x, y \in C ;
$$

(ii) uniformly $\Theta$ - Lipschitzian if there exists $\Theta>0$ such that

$$
\left\|T^{n} x-T^{n} y\right\| \leqslant \Theta\|x-y\|, \forall x, y \in C, \forall n \geqslant 1 ;
$$


(iii) pseudo-contractive if

$$
\|\mathrm{T} x-\mathrm{T} y\|^{2} \leqslant\|x-y\|^{2}+\|(I-T) x-(I-T) y\|^{2}, \forall x, y \in C ;
$$

(iv) $k$-strict pseudo contractive, if there exists $k \in[0,1)$ such that

$$
\|T x-T y\|^{2} \leqslant\|x-y\|^{2}+k\|(I-T) x-(I-T) y\|^{2}, \forall x, y \in C ;
$$

(v) $\left(k,\left\{\lambda_{n}\right\},\left\{\mu_{n}\right\}, \phi\right)$-total asymptotically strictly pseudo contraction (see [35]), if there exists a constant $k \in[0,1)$ and sequences of nonnegative real numbers $\left\{\lambda_{n}\right\},\left\{\mu_{n}\right\}$, and a strictly increasing continuous function $\phi: \mathbb{R}^{+} \rightarrow \mathbb{R}^{+}$with $\phi(0)$ such that

$$
\left\|T^{n} x-T^{n} y\right\|^{2} \leqslant\|x-y\|^{2}+k\left\|\left(I-T^{n}\right) x-\left(I-T^{n}\right) y\right\|^{2}+\lambda_{n} \phi(\|x-y\|)+\mu_{n}, \forall x, y \in C, \forall n \geqslant 1 .
$$

Let $\mathrm{E}$ be a smooth Banach space, $\mathrm{C}$ a nonempty subset of $\mathrm{E}$, and $\mathrm{T}: \mathrm{C} \rightarrow \mathrm{C}$ a mapping. Following [3], see also [22] we say that $\mathrm{T}$ is of type $(P)$ if

$$
\langle T x-T y, J(x-T x)-J(y-T y)\rangle \geqslant 0, \forall x, y \in C .
$$

If $\mathrm{E}$ is a Hilbert space, then $\mathrm{J}=\mathrm{I}$ and hence $\mathrm{T}$ is of type $(\mathrm{P})$ if and only if $\mathrm{T}$ is firmly nonexpansive, that is,

$$
\|T x-T y\|^{2} \leqslant\langle T x-T y, x-y\rangle, \forall x, y \in C .
$$

For any $x \in H$, there exists a unique nearest point to $C$ denoted by $P_{C} x$, such that

$$
\left\|x-P_{C} x\right\| \leqslant\|x-y\|, \forall x, y \in C .
$$

The mapping $\mathrm{P}_{\mathrm{C}}: \mathrm{H} \rightarrow \mathrm{C}$ is known as the metric projection of $\mathrm{H}$ onto $\mathrm{C}$ and satisfies the following.

\section{Lemma 2.2.}

$$
\left\|y-P_{C} x\right\|^{2}+\left\|x-P_{C} x\right\|^{2} \leqslant\|x-y\|^{2} \forall x \in H, y \in C, \quad\left\langle P_{C} x-x, y-P_{C} x\right\rangle \geqslant 0, \forall y \in C .
$$

For more properties of $\mathrm{P}_{\mathrm{C}}$, one can consult [15].

Definition 2.3 ([15]). A Banach space $E$ is said to have the Kadec-Klee property if whenever $\left\{x_{n}\right\}$ is a sequence in $E$ that converges weakly to $x_{0} \in E$ and $\left\|x_{n}\right\| \rightarrow\left\|x_{0}\right\|$, as $n \rightarrow \infty$, then $\left\{x_{n}\right\}$ converges strongly to $x_{0}$.

Lemma 2.4 ([14]). Let $\mathrm{C}$ be a nonempty subset of a real Hilbert space $\mathrm{H}$ and let $\mathrm{S}: \mathrm{C} \rightarrow \mathrm{C}$ be a uniformly $\Theta$ Lipschitzian and $\left(k,\left\{\lambda_{n}\right\},\left\{\mu_{n}\right\}, \phi\right)$-total asymptotically strictly pseudo contraction, then $S$ is demiclosed at origin. That is, if for any sequence $\left\{x_{n}\right\}$ in $C$ with $x_{n} \rightarrow x$ and $\left\|x_{n}-S x_{n}\right\| \rightarrow 0, n \rightarrow \infty$, we have $x=S x$.

Lemma 2.5. The following statements hold in a real Hilbert space, $\mathrm{H}$.

(i) $\|x+y\|^{2}=\|x\|^{2}+2\langle x, y\rangle+\|y\|^{2}, \forall x, y \in H$;

(ii) $\|x+y\|^{2} \leqslant\|x\|^{2}+\langle y, x+y\rangle, \forall x, y \in H$;

(iii) $\|\alpha x+(1-\alpha) y\|^{2}=\alpha\|x\|^{2}+(1-\alpha)\|y\|^{2}-\alpha(1-\alpha)\|x-y\|^{2}, \forall x, y \in H,, \forall \alpha \in[0,1]$.

Definition $2.6([6,17])$. Let $f: C \times C \rightarrow \mathbb{R}$ be an equilibrium bifunction. That is: $f(x, x)=0$. The equilibrium problem is: find $x^{*} \in C$ :

$$
f\left(x^{*}, y\right) \geqslant 0, \forall y \in C .
$$

We shall denote by $\operatorname{EP}(f)$ the set of solutions of (2.3).

Assumption 2.7. For solving the equilibrium problem, we assume that $f$ and the set $C$ satisfy the following conditions: 
(A1) $f(x, x)=0$ for all $x \in C$;

(A2) $f$ is monotone, that is $f(x, y)+f(y, x) \leqslant 0$ for all $x, y \in C$;

(A3) $f$ is upper hemicontinuous, i.e., for each $x, y, z \in C, \lim _{t \rightarrow 0} f(t z+(1-t) x, y) \leqslant f(x, y)$;

(A4) $f(x,$.$) is convex and lower semi-continuous for each x \in C$.

With the above assumptions, we have the following Lemma.

Lemma 2.8 (Blum and Oettli [6], Combettes and Hirstoaga [17]). Let $\mathrm{C}$ be a nonempty closed and convex subset of a real Hilbert space $\mathrm{H}$. Let $\mathrm{f}: \mathrm{C} \times \mathrm{C} \rightarrow \mathfrak{R}$ be a bifunction satisfying assumptions (A1)-(A4). For $\mathrm{r}>0$ and $x \in \mathrm{H}$, there exists $z \in \mathrm{C}$ such that

$$
f(z, y)+\frac{1}{r}\langle y-z, z-x\rangle \geqslant 0, \forall y \in C .
$$

Moreover, define a mapping $\mathrm{T}_{\mathrm{r}}^{\mathrm{f}}: \mathrm{H} \rightarrow \mathrm{C}$ as follows:

$$
\mathrm{T}_{\mathrm{r}}^{\mathrm{f}}(\mathrm{x})=\left\{z \in \mathrm{C}: \mathrm{f}(z, \mathrm{y})+\frac{1}{\mathrm{r}}\langle\mathrm{y}-z, z-x\rangle \geqslant 0, \mathrm{y} \in \mathrm{C}\right\}
$$

for all $x \in \mathrm{H}$. Then the following conclusions hold:

(1) for each $\mathrm{x} \in \mathrm{H}, \mathrm{T}_{\mathrm{r}}^{\mathrm{f}}(\mathrm{x}) \neq \emptyset$;

(2) $\mathrm{T}_{\mathrm{r}}^{\mathrm{f}}$ is single-valued;

(3) $\mathrm{T}_{\mathrm{r}}^{\mathrm{f}}$ is firmly nonexpansive, i.e., for each $\mathrm{x}, \mathrm{y} \in \mathrm{H}$,

$$
\left\langle T_{r}^{f} x-T_{r}^{f} y, T_{r}^{f} x-T_{r}^{f} y\right\rangle \leqslant\left\langle T_{r}^{f} x-T_{r}^{f} y, x-y\right\rangle ;
$$

(4) $\mathrm{F}\left(\mathrm{T}_{\mathrm{r}}^{\mathrm{f}}\right)=\mathrm{EP}(\mathrm{f})$;

(5) $\mathrm{EP}(\mathrm{f})$ is closed and convex.

\section{Main results}

We now prove our main result.

Theorem 3.1. Let $\mathrm{H}_{1}$ and $\mathrm{H}_{2}$ be two real Hilbert spaces and let $\mathrm{C} \subseteq \mathrm{H}_{1}$ and $\mathrm{Q} \subseteq \mathrm{H}_{2}$ be nonempty closed convex subsets of $\mathrm{H}_{1}$ and $\mathrm{H}_{2}$, respectively. Let $\mathrm{f}: \mathrm{C} \times \mathrm{C} \rightarrow \mathbb{R}$ and $\mathrm{g}: \mathrm{Q} \times \mathrm{Q} \rightarrow \mathbb{R}$ be two bifunctions satisfying assumptions (2.7). Let $\mathrm{S}: \mathrm{C} \rightarrow \mathrm{C}$ be a uniformly $\Theta$ - Lipschitzian and continuous total asymptotically strict pseudo contractive mapping and let $\mathrm{A}: \mathrm{H}_{1} \rightarrow \mathrm{H}_{2}$ be a bounded linear map with adjoint $\mathrm{A}^{*}: \mathrm{H}_{2} \rightarrow \mathrm{H}_{1}$. Let $\Omega:=\{z \in \mathrm{C}: z \in \operatorname{EP}(\mathrm{f}): \mathrm{A} z \in \operatorname{EP}(\mathrm{g})\} \neq \emptyset$ and assume that $\Gamma:=\mathrm{F}(\mathrm{S}) \cap \Omega \neq \emptyset$. Let the stepsize $\gamma^{\mathrm{k}}$ be chosen such that, for some $\epsilon>0, \gamma^{k} \in\left(\epsilon, \min \left\{\frac{2\left\|A w^{k}-T_{s_{k}}^{g} A w^{k}\right\|^{2}}{\left\|A^{*}\left(A w^{k}-T_{r_{k}}^{g} A w^{k}\right)\right\|^{2}}-\epsilon, \frac{\left\|A w^{k}-T_{s_{k}}^{g} A w^{k}\right\|^{2}}{2\left\|A^{*}\left(A w^{k}-T_{r_{k}}^{g} A w^{k}\right)\right\|^{2}}-\epsilon\right\}\right)$ if $w^{k} \neq T_{s_{k}}^{g} A w^{k}$, otherwise $\gamma^{k}=\gamma$ (where $\gamma$ is any nonnegative constant). For arbitrary $z^{0}, z^{1} \in \mathrm{H}_{1}$, define the sequence $\left\{z^{k}\right\}$ by the following algorithm:

$$
\left\{\begin{array}{l}
\mathrm{C}_{0}=\mathrm{H}_{1}, \\
w^{k}=z^{k}+\left(z^{k}-z^{k-1}\right), \\
u^{k}=T_{r_{k}}^{f}\left(w^{k}-\gamma^{k} A^{*}\left(I-T_{s_{k}}^{g}\right) A w^{k}\right), \\
v^{k}=\alpha_{k} u^{k}+\left(1-\alpha_{k}\right) S^{k} u^{k}, \\
C_{k+1}=\left\{q \in C_{k}:\left\|v^{k}-q\right\|^{2} \leqslant\left\|w^{k}-q\right\|^{2}+\theta^{k}\right\}, \\
z^{k+1}=P_{C_{k+1}} z^{0}, \forall k \geqslant 1,
\end{array}\right.
$$

where $\theta^{k}:=\left(1-\alpha_{k}\right)\left\{\lambda_{k} \phi(M)+\lambda_{k} M^{*} D_{k}+\mu_{k}\right\}$ with $D_{k}=\sup \left\{\left\|w^{k}-p\right\| ; p \in \Gamma\right\},\left\{r_{k}\right\},\left\{s_{k}\right\}$ are two sequences of positive real numbers and $\left\{\alpha_{k}\right\}$ is a sequence in $(0,1)$ such that the following conditions are satisfied:

(I) $0 \leqslant k<a \leqslant \alpha_{k} \leqslant b<1$; 
(II) $\liminf _{k \rightarrow \infty} r_{k}>0$, $\liminf _{k \rightarrow \infty} s_{k}>0$;

(III) $\sum_{k=1}^{\infty} \mu_{k}<\infty$ and $\sum_{k=1}^{\infty} \lambda_{k}<\infty$;

(IV) there exist constants $M, M^{*}>0$ such that $\phi(\lambda) \leqslant M^{*} \lambda^{2}, \forall \lambda \geqslant M$.

Then the sequence $\left\{z^{\mathrm{k}}\right\}$ generated by (3.1) converges strongly to $\zeta \in \Gamma$, with $\zeta=\mathrm{P}_{\Gamma} z^{0}$.

Proof. We divide the proof into six steps.

Step I: We show that the sequence $\left\{z^{k}\right\}$ defined in (3.1) is well defined. For this, we first show by mathematical induction that $\Gamma \subseteq \mathrm{C}_{k}, \forall \mathrm{k} \geqslant 1$. Obviously $\Gamma \subseteq \mathrm{C}_{0}=\mathrm{H}_{1}$. Now, assume that $\Gamma \subseteq \mathrm{C}_{\mathrm{j}}$ for some $\mathrm{j} \geqslant 1$. Let $p \in \Gamma$, from (3.1) and (2.1), we estimate as follows:

$$
\begin{aligned}
\left\|u^{j}-p\right\|^{2}= & \left\|T_{r_{j}}^{f}\left(w^{j}-\gamma^{j} A^{*}\left(I-T_{s_{j}}^{g}\right) A w^{j}\right)-T_{r_{j}}^{f} p\right\|^{2} \\
\leqslant & \left.\| w^{j}-\gamma^{j} A^{*}\left(I-T_{s_{j}}^{g}\right) A w^{j}\right)-p \|^{2} \\
= & \left\|w^{j}-p\right\|^{2}-2 \gamma^{j}\left\langle w^{j}-p, A^{*}\left(I-T_{s_{j}}^{g}\right) A w^{j}\right\rangle+\left(\gamma^{j}\right)^{2}\left\|A^{*}\left(I-T_{s_{j}}^{g}\right) A w^{j}\right\|^{2} \\
= & \left\|w^{j}-p\right\|^{2}-2 \gamma^{j}\left\langle A w^{j}-A p,\left(I-T_{s_{j}}^{g}\right) A w^{j}\right\rangle+\left(\gamma^{j}\right)^{2}\left\|A^{*}\left(I-T_{s_{j}}^{g}\right) A w^{j}\right\|^{2} \\
= & \left\|w^{j}-p\right\|^{2}-2 \gamma^{j}\left\langle A w^{j}-T_{s_{j}}^{g} A w^{j}+T_{s_{j}}^{g} A w^{j}-A p,\left(I-T_{s_{j}}^{g}\right) A w^{j}\right\rangle \\
& +\left(\gamma^{j}\right)^{2}\left\|A^{*}\left(I-T_{s_{j}}^{g}\right) A w^{j}\right\|^{2} \\
= & \left\|w^{j}-p\right\|^{2}-2 \gamma^{j}\left\|A w^{j}-T_{s_{j}}^{g} A w^{j}\right\|^{2}-2 \gamma^{j}\left\langle T_{s_{j}}^{g} A w^{j}-T_{s_{j}}^{g} A p,\left(I-T_{s_{j}}^{g}\right) A w^{j}\right\rangle \\
& +\left(\gamma^{j}\right)^{2}\left\|A^{*}\left(I-T_{s_{j}}^{g}\right) A w^{j}\right\|^{2} \\
\leqslant & \left\|w^{j}-p\right\|^{2}-2 \gamma^{j}\left\|A w^{j}-T_{s_{j}}^{g} A w^{j}\right\|^{2}+\left(\gamma^{j}\right)^{2}\left\|A^{*}\left(I-T_{s_{j}}^{g}\right) A w^{j}\right\|^{2} u \operatorname{sing}(2.1) \\
= & \left\|w^{j}-p\right\|^{2}-\gamma^{j}\left(2\left\|A w^{j}-T_{s_{j}}^{g} A w^{j}\right\|^{2}-\gamma^{j}\left\|A^{*}\left(I-T_{s_{j}}^{g}\right) A w^{j}\right\|^{2}\right) \\
\leqslant & \left\|w^{j}-p\right\|^{2} .
\end{aligned}
$$

That is.

$$
\left\|u^{j}-p\right\|^{2} \leqslant\left\|w^{j}-p\right\|^{2} .
$$

From (3.1) and utilizing (3.3), we have the following:

$$
\begin{aligned}
\left\|v^{j}-p\right\|^{2}= & \left\|\alpha_{j} u^{j}+\left(1-\alpha_{j}\right) S^{j} u^{j}-p\right\|^{2} \\
= & \alpha_{j}\left\|u^{j}-p\right\|^{2}+\left(1-\alpha_{j}\right)\left\|S^{j} u^{j}-p\right\|^{2}-\alpha_{j}\left(1-\alpha_{j}\right)\left\|u^{j}-S^{j} u^{j}\right\|^{2} \\
\leqslant & \alpha_{j}\left\|u^{j}-p\right\|^{2}+\left(1-\alpha_{j}\right)\left\{\left\|u^{j}-p\right\|^{2}+\kappa\left\|u^{j}-S^{j} u^{j}\right\|^{2}+\lambda_{j} \phi\left(\left\|u^{j}-p\right\|\right)+\mu_{j}\right\} \\
& -\alpha_{j}\left(1-\alpha_{j}\right)\left\|u^{j}-S^{j} u^{j}\right\|^{2} \\
\leqslant & \left\|u^{j}-p\right\|^{2}+\left(1-\alpha_{j}\right)\left\{k\left\|u^{j}-S^{j} u^{j}\right\|^{2}+\lambda_{j} \phi(M)+\lambda_{j} M^{*}\left\|u^{j}-p\right\|^{2}+\mu_{j}\right\} \\
& -\alpha_{j}\left(1-\alpha_{j}\right)\left\|u^{j}-S^{j} u^{j}\right\|^{2} \\
= & \left\|u^{j}-p\right\|^{2}-\left(\alpha_{j}-\kappa\right)\left(1-\alpha_{j}\right)\left\|u^{j}-S^{j} u^{j}\right\|^{2}+\left(1-\alpha_{j}\right)\left\{\lambda_{j} \phi(M)+\lambda_{j} M^{*}\left\|u^{j}-p\right\|^{2}+\mu_{j}\right\} .
\end{aligned}
$$

From condition (I), we have that $\left(\alpha_{j}-\kappa\right)>0$ and so we conclude that

$$
\left\|v^{j}-p\right\|^{2} \leqslant\left\|u^{j}-p\right\|^{2}+\theta^{j},
$$

where $\theta^{j}=\left(1-\alpha_{j}\right)\left\{\lambda_{j} \phi(M)+\lambda_{j} M^{*} D_{j}+\mu_{j}\right\}$ with $D_{j}:=\sup \left\{\left\|w^{j}-p\right\|^{2}: p \in \Gamma\right\}$. From (3.4) we obtain that $p \in C_{j+1}$. Since $p$ is arbitrary, we conclude that $\Gamma \subseteq C_{k+1}, \forall k \geqslant 1$. Next we show that the set $C_{k}$ is closed and convex for all $k \geqslant 1$. Observe that

$$
\left\{z \in C_{k}:\left\|u^{k}-z\right\|^{2} \leqslant\left\|w^{k}-z\right\|^{2}+\theta^{k}\right\}=\left\{z \in C_{k}:\left\|u^{k}\right\|^{2}-\left\|w^{k}\right\|^{2} \leqslant 2\left\langle u^{k}-w^{k}, z\right\rangle+\theta^{k}\right\} .
$$

So, the set $C_{k}$ is closed and convex for all $k \geqslant 1$. Hence the sequence $\left\{z^{k}\right\}$ defined by (3.1) is well defined. 
Step II: We show that $\left\{z^{k}\right\},\left\{v^{k}\right\},\left\{u^{k}\right\}$, and $\left\{w^{k}\right\}$ are all bounded.

Since $z^{k}=P_{C_{k}} z^{0}$, then using (2.2), we have

$$
0 \leqslant\left\langle z^{\mathrm{k}}-z^{0}, z^{*}-z^{\mathrm{k}}\right\rangle, \forall z^{*} \in \mathrm{C}_{\mathrm{k}} .
$$

For each $p \in \Gamma$, we get

$$
\begin{aligned}
0 \leqslant\left\langle z^{\mathrm{k}}-z^{0}, \mathrm{p}-z^{\mathrm{k}}\right\rangle & =\left\langle z^{\mathrm{k}}-z^{0}, \mathrm{p}+z^{0}-z^{0}-z^{\mathrm{k}}\right\rangle \\
& =\left\langle z^{\mathrm{k}}-z^{0}, z^{0}-z^{\mathrm{k}}\right\rangle+\left\langle z^{\mathrm{k}}-z^{0}, \mathrm{p}-z^{0}\right\rangle \leqslant-\left\|z^{\mathrm{k}}-z^{0}\right\|^{2}+\left\|z^{\mathrm{k}}-z^{0} \mid\right\|\left\|\mathrm{p}-z^{0}\right\| .
\end{aligned}
$$

From (3.5), we get

$$
\left\|z^{k}-z^{0}\right\| \leqslant\left\|p-z^{0}\right\|, \forall p \in \Gamma, \forall k \geqslant 1 .
$$

Hence $\left\{z^{k}\right\}$ is bounded. Consequently $\left\{v^{k}\right\},\left\{u^{k}\right\}$, and $\left\{w^{k}\right\}$ are all bounded.

Step III: $\lim _{k \rightarrow \infty}\left\|A w^{k}-T_{s_{k}}^{g} A w^{k}\right\|=0$.

From $z^{\mathrm{k}}=\mathrm{P}_{\mathrm{C}_{\mathrm{k}}} z^{0}$ and $z^{\mathrm{k}+1}=\mathrm{P}_{\mathrm{C}_{\mathrm{k}+1}} z^{0} \in \mathrm{C}_{\mathrm{k}+1} \subseteq \mathrm{C}_{\mathrm{k}}$, we have

$$
0 \leqslant\left\langle z^{k}-z^{0}, z^{k+1}-z^{k}\right\rangle \text {. }
$$

Following similar argument as in (3.5), we have

$$
\left\|z^{k}-z^{0}\right\| \leqslant\left\|z^{k+1}-z^{0}\right\|, \forall k \geqslant 1 .
$$

From (3.6) and (3.7), we conclude that $\left\{\left\|z^{k}-z^{0}\right\|\right\}$ is bounded and nondecreasing. Hence

$$
\lim _{k \rightarrow \infty}\left\|z^{k}-z^{0}\right\| \text { exists. }
$$

Furthermore, since $z^{m}=P_{C_{m}} z^{0} \in C_{m} \subset C_{k}$ for $m>k$, we obtain from Lemma 2.2 that

$$
\left\|z^{\mathrm{m}}-z^{\mathrm{k}}\right\|^{2} \leqslant\left\|z^{\mathrm{k}}-z^{0}\right\|^{2}-\left\|z^{\mathrm{k}}-z^{0}\right\|^{2} .
$$

Since $\lim _{n \rightarrow \infty}\left\|z^{k}-z^{0}\right\|$ exists, we have from (3.8) that

$$
\lim _{n \rightarrow \infty}\left\|z^{m}-z^{k}\right\|=0
$$

Thus, $\left\{z^{k}\right\}$ is a Cauchy sequence. Hence

$$
\lim _{k \rightarrow \infty}\left\|z^{k+1}-z^{k}\right\|=0 .
$$

Since $z^{k+1} \in C_{k+1}$, then

$$
\left\|v^{k}-z^{k+1}\right\| \leqslant\left\|w^{k}-z^{k+1}\right\|+\sqrt{\theta^{k}}
$$

Notice that

$$
\left\|w^{k}-z^{k}\right\|=\left\|z^{k}-z^{k-1}\right\| \rightarrow 0 k \rightarrow \infty \text { from (3.9). }
$$

Also,

$$
\left\|w^{k}-z^{k+1}\right\|=\left\|w^{k}-z^{k}+z^{k}-z^{k+1}\right\| \leqslant\left\|w^{k}-z^{k}\right\|+\left\|z^{k}-z^{k+1}\right\| \rightarrow 0 \text { by (3.9). }
$$

Applying (3.11) in (3.10), we have that

$$
\lim _{k \rightarrow \infty}\left\|v^{k}-z^{k+1}\right\|=0
$$

Therefore, by (3.11) and (3.12)

$$
\left\|v^{k}-w^{k}\right\| \leqslant\left\|v^{k}-z^{k+1}\right\|+\left\|z^{k+1}-w^{k}\right\| \rightarrow 0 k \rightarrow \infty .
$$


From (3.2), we have

$$
\begin{aligned}
\gamma_{k}\left(2\left\|A w^{k}-T_{s_{k}}^{g} A w^{k}\right\|^{2}-\gamma_{k}\left\|A^{*}\left(I-T_{s_{k}}^{g}\right) A w^{k}\right\|^{2}\right. & \leqslant\left\|w^{k}-p\right\|^{2}-\left\|u^{k}-p\right\|^{2} \\
& \leqslant\left\|w^{k}-p\right\|^{2}-\left\|v^{k}-p\right\|^{2}+\theta^{k} \\
& \leqslant\left(\left\|w^{k}-p\right\|+\left\|v^{k}-p\right\|\right)\left\|w^{k}-v^{k}\right\|+\theta^{k}
\end{aligned}
$$

Hence,

$$
\begin{aligned}
2 \gamma_{k}\left\|A w^{k}-T_{s_{k}}^{g} A w^{k}\right\|^{2} \leqslant & \left(\left\|w^{k}-p\right\|+\left\|v^{k}-p\right\|\right)\left\|w^{k}-v^{k}\right\|+\theta^{k}+\gamma_{k}^{2}\left\|A^{*}\left(I-T_{s_{k}}^{g}\right) A w^{k}\right\|^{2}, \\
2\left\|A w^{k}-T_{s_{k}}^{g} A w^{k}\right\|^{2} \leqslant & \frac{1}{\gamma_{k}}\left\{\left(\left\|w^{k}-p\right\|+\left\|v^{k}-p\right\|\right)\left\|w^{k}-v^{k}\right\|+\theta^{k}\right\}+\gamma_{k}\left\|A^{*}\left(I-T_{s_{k}}^{g}\right) A w^{k}\right\|^{2} \\
\leqslant & \frac{1}{\gamma_{k}}\left\{\left(\left\|w^{k}-p\right\|+\left\|v^{k}-p\right\|\right)\left\|w^{k}-v^{k}\right\|+\theta^{k}\right\} \\
& +\frac{\left\|\left(I-T_{s_{k}}^{g}\right) A w^{k}\right\|^{2}}{2\left\|A^{*}\left(I-T_{s_{k}}^{g}\right) A w^{k}\right\|^{2}}\left\|A^{*}\left(I-T_{s_{k}}^{g}\right) A w^{k}\right\|^{2}, \\
4\left\|A w^{k}-T_{s_{k}}^{g} A w^{k}\right\|^{2} \leqslant & \frac{2}{\gamma_{k}}\left\{\left(\left\|w^{k}-p\right\|+\left\|v^{k}-p\right\|\right)\left\|w^{k}-v^{k}\right\|+\theta^{k}\right\}+\left\|\left(I-T_{s_{k}}^{g}\right) A w^{k}\right\|^{2} .
\end{aligned}
$$

So, using (3.14), we have

$$
3\left\|A w^{k}-T_{s_{k}}^{g} A w^{k}\right\|^{2} \leqslant \frac{2}{\gamma_{k}}\left\{\left(\left\|w^{k}-p\right\|+\left\|v^{k}-p\right\|\right)\left\|w^{k}-v^{k}\right\|+\theta^{k}\right\} \rightarrow 0 .
$$

That is

$$
\lim _{k \rightarrow \infty}\left\|A w^{k}-T_{s_{k}}^{g} A w^{k}\right\|=0
$$

Step IV: $\lim \left\|w^{k}-S w^{k}\right\|=0$.

Let $p \in \Gamma$. Using firm nonexpansiveness of $T_{r_{k}}^{f}$, we have the following estimates:

$$
\begin{aligned}
\left\|u^{k}-p\right\|^{2}= & \left\|T_{r_{k}}^{f}\left(w^{k}-\gamma_{k} A^{*}\left(I-T_{s_{k}}^{g}\right) A w^{k}\right)-T_{r_{k}}^{f} p\right\|^{2} \\
\leqslant & \left\langle u^{k}-p, w^{k}-\gamma_{k} A^{*}\left(I-T_{s_{k}}^{g}\right) A w^{k}-p\right\rangle \\
= & \frac{1}{2}\left\{\left\|u^{k}-p\right\|^{2}+\left\|w^{k}-\gamma_{k} A^{*}\left(I-T_{s_{k}}^{g}\right) A w^{k}-p\right\|^{2}-\left\|u^{k}-w^{k}-\gamma_{k} A^{*}\left(I-T_{s_{k}}^{g}\right) A w^{k}\right\|^{2}\right\} \\
\leqslant & \frac{1}{2}\left\{\left\|u^{k}-p\right\|^{2}+\left\|w^{k}-p\right\|^{2}-\left\|u^{k}-w^{k}-\gamma_{k} A^{*}\left(I-T_{s_{k}}^{g}\right) A w^{k}\right\|^{2}\right\} \\
= & \frac{1}{2}\left\{\left\|u^{k}-p\right\|^{2}+\left\|w^{k}-p\right\|^{2}-\left(\left\|u^{k}-w^{k}\right\|^{2}+\gamma_{k}^{2}\left\|A^{*}\left(I-T_{s_{k}}^{g}\right) A w^{k}\right\|^{2}\right.\right. \\
& \left.\left.-2 \gamma_{k}\left\langle u^{k}-w^{k}, A^{*}\left(I-T_{s_{k}}^{g}\right) A w^{k}\right\rangle\right)\right\} \\
\leqslant & \left\|w^{k}-p\right\|^{2}-\left\|u^{k}-w^{k}\right\|^{2}+2 \gamma_{k}\left\|A\left(u^{k}-w^{k}\right)\right\|\left\|A^{*}\left(I-T_{s_{k}}^{g}\right) A w^{k}\right\| .
\end{aligned}
$$

Notice that from argument of (3.3), we have

$$
\left\|v^{k}-p\right\|^{2} \leqslant \alpha_{k}\left\|w^{k}-p\right\|^{2}+\left(1-\alpha_{k}\right)\left\|u^{k}-p\right\|^{2}+\theta^{k} .
$$

Applying (3.17) in (3.16) and re-arranging the terms, we have

$$
\left(1-\alpha_{k}\right)\left\|u^{k}-w^{k}\right\|^{2} \leqslant\left(\left\|w^{k}-p\right\|+\left\|v^{k}-p\right\|\right)\left\|v^{k}-w^{k}\right\|+2 \gamma^{k}\left\|A ( u ^ { k } - w ^ { k } ) \left|\left\|\mid A^{*}\left(I-T_{s_{k}}^{g}\right) A w^{k}\right\| .\right.\right.
$$

Letting $k \rightarrow \infty$, we have using (3.13) and (3.15) that

$$
\left\|u^{k}-w^{k}\right\| \rightarrow 0, k \rightarrow \infty .
$$


Furthermore, from (3.13) and (3.18) we have

$$
\left\|v^{k}-w^{k}\right\| \leqslant\left\|v^{k}-u^{k}\right\|+\left\|u^{k}-w^{k}\right\| \rightarrow 0, k \rightarrow \infty .
$$

From (3.1), we get that $\left\|v^{k}-u^{k}\right\|=\left(1-\alpha_{k}\right)\left\|S^{k} u^{k}-u^{k}\right\|$. Utilizing condition (I) and conclusion (3.13), we obtain

$$
\left|S^{k} u^{k}-u^{k}\right| \mid \rightarrow 0, k \rightarrow \infty
$$

Using (3.19) we obtain

$$
\begin{gathered}
\left\|S^{k} u^{k}-w^{k}\right\| \leqslant\left\|S^{k} u^{k}-u^{k}\right\|+\left\|u^{k}-w^{k}\right\| \rightarrow 0, k \rightarrow \infty \\
\left\|S^{k} u^{k}-v^{k}\right\| \leqslant\left\|S^{k} u^{k}-u^{k}\right\|+\left\|u^{k}-v^{k}\right\| \rightarrow 0, k \rightarrow \infty .
\end{gathered}
$$

Utilizing (3.20), we get

$$
\begin{aligned}
\left\|S^{k} w^{k}-w^{k}\right\| & \leqslant\left\|S^{k} w^{k}-S^{k} u^{k}\right\|+\left\|S^{k} u^{k}-w^{k}\right\| \leqslant \Theta\left\|w^{k}-u^{k}\right\|+\left\|S^{k} u^{k}-w^{k}\right\| \rightarrow 0, k \rightarrow \infty . \\
\left\|w^{k}-S w^{k}\right\| & \leqslant\left\|w^{k}-S^{k} w^{k}\right\|+\left\|S^{k} w^{k}-S w^{k}\right\| \leqslant\left\|w^{k}-S^{k} w^{k}\right\|+\Theta\left\|S^{k-1} w^{k}-w^{k}\right\| \rightarrow 0, k \rightarrow \infty .
\end{aligned}
$$

Step V: $\omega\left(w^{k}\right) \subseteq \Gamma$, where $\omega\left(w^{k}\right)$ is the set of all weak cluster points of $\left\{w^{k}\right\}$.

Since $\left\{w^{k}\right\}$ is bounded, $\omega\left(w^{k}\right) \neq \emptyset$. Let $\zeta \in \omega\left(w^{k}\right)$, then there exists a subsequence $\left\{w^{k_{i}}\right\}$ of $\left\{w^{k}\right\}$ such that $w^{k_{i}} \rightarrow \zeta$. Applying Lemma 2.4 and conclusion (3.21), we get that $\zeta \in F(S)$. Next, we verify that $\zeta \in \operatorname{EP}(f)$. From definition of $\left\{u^{k}\right\}, u^{k}=T_{r_{k}}^{f}\left(w^{k}-\gamma_{k} A^{*}\left(I-T_{s_{k}}^{g}\right) A w^{k}\right)$. That is:

$$
f\left(u^{k}, y\right)+\frac{1}{r_{k}}\left\langle y-u^{k}, u^{k}-w^{k}-\gamma_{k} A^{*}\left(I-T_{s_{k}}^{g}\right) A w^{k}\right\rangle \geqslant 0, \forall y \in C .
$$

Replacing $k$ with $k_{i}$, we have

$$
f\left(u^{k_{i}}, y\right)+\frac{1}{r_{k_{i}}}\left\langle y-u^{k_{i}}, u^{k_{i}}-w^{k_{i}}\right\rangle-\frac{1}{r_{k_{i}}}\left\langle y-u^{k_{i}}, \gamma_{k} A^{*}\left(I-T_{s_{k_{i}}}^{g}\right) A w^{k_{i}}\right\rangle \geqslant 0 .
$$

That is

$$
\frac{1}{r_{k_{i}}}\left\langle y-u^{k_{i}}, u^{k_{i}}-w^{k_{i}}\right\rangle-\frac{1}{r_{k_{i}}}\left\langle y-u^{k_{i}}, \gamma_{k_{i}} A^{*}\left(I-T_{s_{k_{i}}}^{g}\right) A w^{k_{i}}\right\rangle \geqslant f\left(y, u^{k_{i}}\right) .
$$

By (A4), (3.22), (3.15), (3.18), and taking limit as $k \rightarrow \infty$, we have

$$
f(y, \zeta) \leqslant 0, \quad \forall y \in H_{1} .
$$

Let $z_{\mathrm{t}}=\mathrm{t} \mathrm{y}+(1-\mathrm{t}) \zeta \quad \forall \mathrm{t} \in(0,1]$ and $\mathrm{y} \in \mathrm{C}$. This implies that $z_{\mathrm{t}} \in \mathrm{C}$. Hence, $\mathrm{f}\left(z_{\mathrm{t}}, \zeta\right) \leqslant 0$. From (A1),

$$
0 \leqslant f\left(z_{t}, z_{t}\right) \leqslant \operatorname{tf}\left(z_{t}, y\right)+(1-t) f\left(z_{t}, \zeta\right) \leqslant t f\left(z_{t}, y\right)
$$

Hence,

$$
0 \leqslant f\left(z_{\mathrm{t}}, \mathrm{y}\right)
$$

from condition (A3), we obtain

$$
f(\zeta, y) \geqslant 0 \quad \forall y \in H_{1}
$$

This implies that

$$
\zeta \in \operatorname{EP}(f)
$$

Since $w^{k_{i}} \rightarrow \zeta$ and $A$ is a bounded linear operator, then $A w^{k_{i}} \rightarrow A \zeta$. Hence, it follows from (3.15) that

$$
\mathrm{T}_{\mathrm{s}_{\mathrm{i}}}^{\mathrm{g}} \mathrm{A} w^{\mathrm{k}_{\mathrm{i}}} \rightarrow A \zeta, i \rightarrow \infty
$$


From Lemma 2.5, we have

$$
g\left(T_{s_{k_{i}}}^{g} A w^{k_{i}}, y\right)+\frac{1}{s_{k_{i}}}\left\langle y-T_{s_{k_{i}}}^{g} A w^{k_{i}}, T_{s_{k_{i}}}^{g} A w^{k_{i}}-A w^{k_{i}}\right\rangle \geqslant 0, \forall y \in Q
$$

Since $g$ is upper-hemicontinuous in the first argument, we have by taking limsup as $i \rightarrow \infty$ in (3.23), condition (II) and (3.15) that

$$
g(A \zeta, y) \geqslant 0, \forall \zeta \in Q
$$

That is, $A \zeta \in \operatorname{EP}(\mathrm{g})$. So $\zeta \in \Gamma$.

Step VI: $z^{\mathrm{k}} \rightarrow \zeta=\mathrm{P}_{\Gamma} z^{0}, k \rightarrow \infty$. Let $\xi=\mathrm{P}_{\Gamma} z^{0}$. Then $\xi \in \Gamma$. From $\left\|z^{\mathrm{k}}-z^{0}\right\| \leqslant\left\|\xi-z^{0}\right\|$, we have

$$
\left\|\xi-z^{0}\right\| \leqslant\left\|\zeta-z^{0}\right\| \leqslant \liminf _{i \rightarrow \infty}\left\|z^{k_{i}}-z^{0}\right\| \leqslant \limsup _{i \rightarrow \infty}\left\|z^{k_{i}}-z^{0}\right\| \leqslant\left\|\xi-z^{0}\right\| .
$$

This implies that $\left\|\xi-z^{0}\right\| \leqslant\left\|\zeta-z^{0}\right\| \leqslant \lim _{i \rightarrow \infty}\left\|z^{k_{i}}-z^{0}\right\| \leqslant\left\|\xi-z^{0}\right\|$. So, $\lim _{i \rightarrow \infty}\left\|z^{k_{i}}-z^{0}\right\|=\left\|\xi-z^{0}\right\|=$ $\left\|\zeta-z^{0}\right\|$. Furthermore, by uniqueness of $P_{\Gamma} z^{0}$, we have that $\xi=\zeta$ and so $\left\|z^{k_{i}}\right\| \rightarrow\|\zeta\|$. Since Hilbert spaces satisfy Kadec-Klee property, we conclude that $z^{k_{i}} \rightarrow \zeta, i \rightarrow \infty$. But $\left\{z^{k}\right\}$ is Cauchy and so it is convergent. Hence,

$$
z^{k} \rightarrow \zeta, k \rightarrow \infty
$$

This completes the proof.

Remark 3.2. In this work, we proved strong convergence theorem for approximating solution of split equilibrium problem and fixed point of a total asymptotically strictly pseudocontractive mapping in real Hilbert spaces. In our algorithm, the stepsize is independent of the knowledge of norm of the bounded linear map, a restrictive condition that has been imposed by many authors (see, e.g., [18, 20, $24,35])$. Furthermore, no compactness-type condition was imposed either on the domain of the operator or on the operator itself as is the case with many similar results in the literature (e.g., [14, 20]). We also incoorporated inertial step into our algorithm which is known to speed up convergence rate of iterative algorithms. Finally, we note that the result of Theorem 3.1 of this manuscript can be extended to finite family of total asymptotically strictly pseudocontractive mappings. We did not prove that since the proof follows same arguments as those used in proving Theorem 3.1 here.

\section{Application}

In this section, we apply the result of Theorem 3.1 to study SEP and fixed point of asymptotically strictly pseudocontractive mapping in real Hilbet spaces.

Definition 4.1. A mapping $\mathrm{T}: \mathrm{C} \rightarrow \mathrm{C}$ is said to be an asymptotically $\mathrm{k}$-strictly pseudocontractive mapping (see $[18,24])$, if there exists a sequence $\left\{v_{n}\right\} \subset[1, \infty)$ with $\lim _{n \rightarrow \infty} v_{n}=1$ and a constant $\lambda \in[0,1)$ such that

$$
\left\|T^{n} x-T^{n} y\right\|^{2} \leqslant v_{n}^{2}\|x-y\|^{2}+\lambda\left\|\left(I-T^{n}\right) x-\left(I-T^{n} y\right)\right\|^{2}, \forall x, y \in C .
$$

Lemma 4.2 ([18]). Assume that $\mathrm{C}$ is a closed and convex subset of a real Hilbert space $\mathrm{H}$, and let $\mathrm{T}: \mathrm{C} \rightarrow \mathrm{C}$ be an asymptotically $\lambda$-strict pseudocontraction with $\operatorname{Fix}(\mathrm{T}) \neq \emptyset$. Then:

(i) for each $\mathrm{n} \geqslant 1, \mathrm{~T}^{\mathrm{n}}$ satisfies the Lipschitz condition

$$
\left\|T^{n} x-T^{n} y\right\| \leqslant L_{n}\|x-y\|, \forall x, y \in C, \text { where } L_{n}=\frac{\lambda+\sqrt{1+\left(k_{n}-1\right)(1-\lambda)}}{1-\lambda} ;
$$

(ii) $\mathrm{T}$ is demiclosed at 0 ;

(iii) $\mathrm{F}(\mathrm{T})$ is closed and convex. 
Theorem 4.3. Let $\mathrm{H}_{1}$ and $\mathrm{H}_{2}$ be two real Hilbert spaces and let $\mathrm{C} \subseteq \mathrm{H}_{1}$ and $\mathrm{Q} \subseteq \mathrm{H}_{2}$ be nonempty closed convex subsets of $\mathrm{H}_{1}$ and $\mathrm{H}_{2}$, respectively. Let $\mathrm{f}: \mathrm{C} \times \mathrm{C} \rightarrow \mathbb{R}$ and $\mathrm{g}: \mathrm{Q} \times \mathrm{Q} \rightarrow \mathbb{R}$ be two bifunctions satisfying assumptions (2.7). Let $\mathrm{S}: \mathrm{C} \rightarrow \mathrm{C}$ be an asymptotically $\mathrm{K}$-strictly pseudocontractive mapping with $(\kappa \in[0,1))$ and $\left\{v_{k}\right\} \subset[1, \infty): v_{k} \rightarrow 1$, and let $A: H_{1} \rightarrow H_{2}$ be a bounded linear map with adjoint $A^{*}: H_{2} \rightarrow H_{1}$. Let $\Omega:=\{z \in \mathrm{C}: z \in \mathrm{EP}(\mathrm{f}): \mathrm{Az} \in \mathrm{EP}(\mathrm{g})\} \neq \emptyset$ and assume that $\Gamma:=\mathrm{F}(\mathrm{S}) \cap \Omega \neq \emptyset$. Let the stepsize $\gamma^{\mathrm{k}}$ be chosen such that, for some $\epsilon>0, \gamma^{k} \in\left(\epsilon, \min \left\{\frac{2\left\|A w^{k}-T_{s_{k}}^{g} A w^{k}\right\|^{2}}{\left\|A^{*}\left(A w^{k}-T_{r_{k}}^{g} A w^{k}\right)\right\|^{2}}-\epsilon, \frac{\left\|A w^{k}-T_{s_{k}}^{g} A w^{k}\right\|^{2}}{2\left\|A^{*}\left(A w^{k}-T_{r_{k}}^{g} A w^{k}\right)\right\|^{2}}-\epsilon\right\}\right)$ if $w^{k} \neq T_{s_{k}}^{g} A w^{k}$, otherwise, $\gamma^{k}=\gamma$ (where $\gamma$ is any nonnegative constant). For arbitrary $z^{0}, z^{1} \in \mathrm{H}_{1}$, define the sequence $\left\{z^{k}\right\}$ by the following algorithm:

$$
\left\{\begin{array}{l}
\mathrm{C}_{0}=\mathrm{H}_{1}, \\
w^{\mathrm{k}}=z^{\mathrm{k}}+\left(z^{\mathrm{k}}-z^{\mathrm{k}-1}\right), \\
\mathrm{u}^{\mathrm{k}}=\mathrm{T}_{\mathrm{r}_{\mathrm{k}}}^{\mathrm{f}}\left(w^{\mathrm{k}}-\gamma^{\mathrm{k}} A^{*}\left(\mathrm{I}-\mathrm{T}_{\mathrm{s}_{\mathrm{k}}}^{\mathrm{g}}\right) A w^{\mathrm{k}}\right), \\
v^{\mathrm{k}}=\alpha_{\mathrm{k}} \mathrm{u}^{\mathrm{k}}+\left(1-\alpha_{\mathrm{k}}\right) \mathrm{S}^{\mathrm{k}} \mathrm{u}^{\mathrm{k}}, \\
\mathrm{C}_{\mathrm{k}+1}=\left\{\mathrm{q} \in \mathrm{C}_{\mathrm{k}}:\left\|v^{\mathrm{k}}-\mathrm{q}\right\|^{2} \leqslant\left\|w^{\mathrm{k}}-\mathrm{q}\right\|^{2}+\theta^{\mathrm{k}}\right\}, \\
z^{\mathrm{k}+1}=\mathrm{P}_{\mathrm{C}_{\mathrm{k}+1}} z^{0}, \forall \mathrm{k} \geqslant 1,
\end{array}\right.
$$

where $\theta^{k}:=\left(1-\alpha_{k}\right)\left(v_{k}^{2}-1\right) D_{k}$ with $D_{k}=\sup \left\{\left\|w^{k}-p\right\|^{2} ; p \in \Gamma\right\},\left\{r_{k}\right\},\left\{s_{k}\right\}$ are two sequences of positive real numbers and $\left\{\alpha_{k}\right\}$ is a sequence in $(0,1)$ such that the following conditions are satisfied:

(I): $0 \leqslant k<a \leqslant \alpha_{k} \leqslant b<1$;

(II): $\lim \inf _{k \rightarrow \infty} r_{k}>0, \quad \liminf _{k \rightarrow \infty} s_{k}>0$.

Then the sequence $\left\{z^{k}\right\}$ generated by (4.1) converges strongly to $\zeta \in \Gamma$, with $\zeta=P_{\Gamma} z^{0}$.

Proof. We divide the proof into six steps.

Step I: We show that $\left\{z^{k}\right\}$ defined in (3.1) is well defined. For this, we first show by mathematical induction that $\Gamma \subseteq \mathrm{C}_{\mathrm{k}}, \forall \mathrm{k} \geqslant 1$. Obviously, $\Gamma \subseteq \mathrm{C}_{0}=\mathrm{H}_{1}$. Now, assume that $\Gamma \subseteq \mathrm{C}_{j}$ for some $j \geqslant 1$. Let $\mathrm{p} \in \Gamma$, from (4.1) and (2.1), we estimate as follows:

$$
\begin{aligned}
\left\|u^{j}-p\right\|^{2} & =\| T_{r_{j}}^{f}\left(w^{j}-\gamma^{j} A^{*}\left(I-T_{s_{j}}^{g} A w^{j}\right)-T_{r_{j}}^{f} p \|^{2}\right. \\
& \leqslant\left\|w^{j}-\gamma^{j} A^{*}\left(I-T_{s_{j}}^{g} A w^{j}\right)-p\right\|^{2} \\
& =\left\|w^{j}-p\right\|^{2}-2 \gamma^{j}\left\langle w^{j}-p, A^{*}\left(I-T_{s_{j}}^{g}\right) A w^{j}\right\rangle+\left(\gamma^{j}\right)^{2}\left\|A^{*}\left(I-T_{s_{j}}^{g}\right) A w^{j}\right\|^{2} \\
& =\left\|w^{j}-p\right\|^{2}-2 \gamma^{j}\left\langle A w^{j}-A p,\left(I-T_{s_{j}}^{g}\right) A w^{j}\right\rangle+\left(\gamma^{j}\right)^{2}\left\|A^{*}\left(I-T_{s_{j}}^{g}\right) A w^{j}\right\|^{2} \\
& =\left\|w^{j}-p\right\|^{2}-2 \gamma^{j}\left\langle A w^{j}-T_{s_{j}}^{g} A w^{j}+T_{s_{j}}^{g} A w^{j}-A p,\left(I-T_{s_{j}}^{g}\right) A w^{j}\right\rangle+\left(\gamma^{j}\right)^{2}\left\|A^{*}\left(I-T_{s_{j}}^{g}\right) A w^{j}\right\|^{2} \\
& =\left\|w^{j}-p\right\|^{2}-2 \gamma^{j}\left\|A w^{j}-T_{s_{j}}^{g} A w^{j}\right\|^{2}-2 \gamma^{j}\left\langle T_{s_{j}}^{g} A w^{j}-T_{s_{j}}^{g} A p,\left(I-T_{s_{j}}^{g}\right) A w^{j}\right\rangle+\left(\gamma^{j}\right)^{2}\left\|A^{*}\left(I-T_{s_{j}}^{g}\right) A w^{j}\right\|^{2} \\
& \leqslant\left\|w^{j}-p\right\|^{2}-2 \gamma^{j}\left\|A w^{j}-T_{s_{j}}^{g} A w^{j}\right\|^{2}+\left(\gamma^{j}\right)^{2}\left\|A^{*}\left(I-T_{s_{j}}^{g}\right) A w^{j}\right\|^{2} u \operatorname{sing}(2.1) \\
& =\left\|w^{j}-p\right\|^{2}-\gamma^{j}\left(2\left\|A w^{j}-T_{s_{j}}^{g} A w^{j}\right\|^{2}-\gamma^{j}\left\|A^{*}\left(I-T_{s_{j}}^{g}\right) A w^{j}\right\|^{2}\right) \\
& \leqslant\left\|w^{j}-p\right\|^{2} .
\end{aligned}
$$

That is.

$$
\left\|u^{j}-p\right\|^{2} \leqslant\left\|w^{j}-p\right\|^{2}
$$

From (4.1) and utilizing (4.2), we have the following:

$$
\begin{aligned}
\left\|v^{j}-p\right\|^{2} & =\left\|\alpha_{j} u^{j}+\left(1-\alpha_{j}\right) S^{j} u^{j}-p\right\|^{2} \\
& =\alpha_{j}\left\|u^{j}-p\right\|^{2}+\left(1-\alpha_{j}\right)\left\|S^{j} u^{j}-p\right\|^{2}-\alpha_{j}\left(1-\alpha_{j}\right)\left\|u^{j}-S^{j} u^{j}\right\|^{2} \\
& \leqslant \alpha_{j}\left\|u^{j}-p\right\|^{2}+\left(1-\alpha_{j}\right)\left\{v_{j}^{2}\left\|u^{j}-p\right\|^{2}+\kappa\left\|u^{j}-S^{j} u^{j}\right\|^{2}\right\}-\alpha_{j}\left(1-\alpha_{j}\right)\left\|u^{j}-S^{j} u^{j}\right\|^{2} \\
& =\alpha_{j}\left\|u^{j}-p\right\|^{2}+\left(1-\alpha_{j}\right)\left\{v_{j}^{2}\left\|u^{j}-p\right\|^{2}+\left\|u^{j}-p\right\|^{2}-\left\|u^{j}-p\right\|^{2}+\kappa\left\|u^{j}-S^{j} u^{j}\right\|^{2}\right\}
\end{aligned}
$$




$$
\begin{aligned}
& -\alpha_{j}\left(1-\alpha_{j}\right)\left\|u^{j}-S^{j} u^{j}\right\|^{2} \\
& =\left\|u^{j}-p\right\|^{2}+\left(1-\alpha_{j}\right)\left(v_{j}^{2}-1\right)\left\|u^{j}-p\right\|^{2}+\left(1-\alpha_{j}\right)\left\{k\left\|u^{j}-S^{j} u^{j}\right\|^{2}\right\}-\alpha_{j}\left(1-\alpha_{j}\right)\left\|u^{j}-S^{j} u^{j}\right\|^{2} \\
& \leqslant\left\|u^{j}-p\right\|^{2}+\left(1-\alpha_{j}\right)\left(v_{j}^{2}-1\right)\left\|u^{j}-p\right\|^{2}+\left(1-\alpha_{j}\right)\left\{k\left\|u^{j}-S^{j} u^{j}\right\|^{2}\right\}-\alpha_{j}\left(1-\alpha_{j}\right)\left\|u^{j}-S^{j} u^{j}\right\|^{2} \\
& =\left\|u^{j}-p\right\|^{2}+\left(1-\alpha_{j}\right)\left(v_{j}^{2}-1\right)\left\|u^{j}-p\right\|^{2}-\left(\alpha_{j}-\kappa\right)\left(1-\alpha_{j}\right)\left\|u^{j}-S^{j} u^{j}\right\|^{2} \text {. }
\end{aligned}
$$

From condition (I), we have that $\left(\alpha_{j}-\kappa\right)>0$, so we conclude that

$$
\left\|v^{j}-p\right\|^{2} \leqslant\left\|u^{j}-p\right\|^{2}+\theta^{j}
$$

where $\theta^{j}:=\left(1-\alpha_{j}\right)\left(v_{j}^{2}-1\right) D_{j}$ with $D_{j}=\sup \left\{\left\|w^{j}-p\right\|^{2} ; p \in \Gamma\right\}$. From (4.3), we obtain that $p \in C_{j+1}$. Since $p$ is arbitrary, we conclude that $\Gamma \subseteq C_{k+1}, \forall k \geqslant 1$. Next, we show that the set $C_{k}$ is closed and convex for all $k \geqslant 1$. Observe that

$$
\left\{z \in C_{k}:\left\|u^{k}-z\right\|^{2} \leqslant\left\|w^{k}-z\right\|^{2}+\theta^{k}\right\}=\left\{z \in C_{k}:\left\|u^{k}\right\|^{2}-\left\|w^{k}\right\|^{2} \leqslant 2\left\langle u^{k}-w^{k}, z\right\rangle+\theta^{k}\right\} .
$$

So, the set $C_{k}$ is closed and convex for all $k \geqslant 1$. Hence the sequence $\left\{z^{k}\right\}$ defined by (3.1) is well defined.

The remaining part of the proof follows same argument as the arguments of steps II-VI of the proof of Theorem 3.1 above. We therefore omit them.

Remark 4.4. Theorem 4.3 carries over easily to the case of finite family of the map, T. We state without proof the result in the next theorem.

Theorem 4.5. Let $\mathrm{H}_{1}$ and $\mathrm{H}_{2}$ be two real Hilbert spaces and let $\mathrm{C} \subseteq \mathrm{H}_{1}$ and $\mathrm{Q} \subseteq \mathrm{H}_{2}$ be nonempty closed convex subsets of $\mathrm{H}_{1}$ and $\mathrm{H}_{2}$, respectively. For $1 \leqslant i \leqslant \mathrm{~N}$, let $\mathrm{f}_{i}: \mathrm{C} \times \mathrm{C} \rightarrow \mathbb{R}$ and $\mathrm{g}_{i}: \mathrm{Q} \times \mathrm{Q} \rightarrow \mathbb{R}$ be two bifunctions satisfying assumptions (2.7). Let $S_{i}: C \rightarrow C$ be an asymptotically $\kappa_{i}$-strictly pseudocontractive mapping, with $\left(\kappa_{i} \in[0,1)\right)$ and $\left\{v_{k}^{i}\right\} \subset[1, \infty): v_{k}^{i} \rightarrow 1, k \rightarrow \infty$ and let $A_{i}: H_{1} \rightarrow H_{2}$ be a bounded linear map with adjoint $A_{i}^{*}: \mathrm{H}_{2} \rightarrow \mathrm{H}_{1}$. Let $\Omega:=\left\{z \in \mathrm{C}: z \in \cap_{i=1}^{N} \operatorname{EP}\left(f_{i}\right): A z \in \cap_{i=1}^{N} \operatorname{EP}\left(g_{i}\right)\right\} \neq$ $\emptyset$ and assume that $\Gamma:=\cap \cap_{i=1}^{N} F\left(S_{i}\right) \cap \Omega \neq \emptyset$. Let the stepsizes $\gamma^{k, i}$ be chosen such that, for some $\epsilon_{i}>0$, $\gamma^{k, i} \in\left(\epsilon_{i}, \min \left\{\frac{2\left\|A w^{k}-T_{s_{k}}^{g_{i}} A w^{k}\right\|^{2}}{\left\|A^{*}\left(A w^{k}-T_{r_{k}}^{g_{i}} A w^{k}\right)\right\|^{2}}-\epsilon_{i}, \frac{\left\|A w^{k}-T_{s_{k}}^{g_{i}} A w^{k}\right\|^{2}}{2\left\|A^{*}\left(A w^{k}-T_{r_{k}}^{g_{i}} A w^{k}\right)\right\|^{2}}-\epsilon_{i}\right\}\right)$ if $w^{k} \neq T_{s_{k}}^{g_{i}} A w^{k}$, otherwise, $\gamma^{k i}=$ $\gamma^{i}$ (where $\gamma^{i}$ is any nonnegative constant), $i=1,2, \ldots, N$. For arbitrary $z^{0}, z^{1} \in \mathrm{H}_{1}$, define the sequence $\left\{z^{k}\right\}$ by the following algorithm:

$$
\left\{\begin{array}{l}
\mathrm{C}_{0}=\mathrm{H}_{1}, \\
w^{k}=z^{k}+\left(z^{k}-z^{k-1}\right), \\
u^{k}=T_{r_{k}}^{f_{i}}\left(w^{k}-\gamma^{k} A_{k(\operatorname{modN})}^{*}\left(I-T_{S_{k}}^{g_{i}}\right) A w^{k}\right), \\
v^{k}=\alpha_{k} u^{k}+\left(1-\alpha_{k}\right) S_{k(\operatorname{modN})}^{k} u^{k}, \\
C_{k+1}=\left\{q \in C_{k}:\left\|v^{k}-q\right\|^{2} \leqslant\left\|w^{k}-q\right\|^{2}+\theta^{k}\right\}, \\
z^{k+1}=P_{C_{k+1}} z^{0}, \forall k \geqslant 1,
\end{array}\right.
$$

where $\theta^{k}:=\left(1-\alpha_{k}\right)\left(v_{k}^{2}-1\right) D_{k}$ with $D_{k}=\sup \left\{\left\|w^{k}-p\right\|^{2} ; p \in \Gamma\right\},\left\{r_{k}\right\},\left\{s_{k}\right\}$ are two sequences of positive real numbers and $\left\{\alpha_{k}\right\}$ is a sequence in $(0,1)$ such that the following conditions are satisfied:

(I): $0 \leqslant k<a \leqslant \alpha_{k} \leqslant b<1$;

(II): $\liminf _{k \rightarrow \infty} r_{k}>0, \quad \liminf _{k \rightarrow \infty} s_{k}>0$.

Then the sequence $\left\{z^{\mathrm{k}}\right\}$ generated by (4.4) converges strongly to $\zeta \in \Gamma$, with $\zeta=\mathrm{P}_{\Gamma} z^{0}$.

Remark 4.6. In [18], the authors proved strong convergence result for common fixed point of finite family of asymptotically strictly pseudocontractive mappings requiring that the domain of the operators be bounded. This is quite restrictive. In Theorem 4.5 of this manuscript, we obtained strong convergence result for a finite family of SEP and common fixed point of finite family of asymptotically strictly pseudocontractive mapping without requiring boundedness of the domain of the mappings. The stepsize used is independent of any knowledge of the norm of the involved bounded linear map. Consequently, our result, Theorem 4.5 is an extension and a significant improvement of the results of [18] and many other important results in this direction of research. 


\section{Acknowledgment}

We thank immensely the referees for their insightful comments that improved the quality of the work.

\section{References}

[1] Y. I. Alber, C. E. Chidume, H. Zegeye, Approximating fixed points of total asymptotically nonexpansive mappings, Fixed Point Theory Appl., 2006 (2006), 20 pages. 1

[2] P. N. Anh, A hybrid extragradient method extended to fixed point problems and equilibrium problems, Optimization, 62 (2013), 271-283. 1

[3] K. Aoyama, F. Kohsaka, W. Takahashi, Three generalizations of firmly nonexpansive mappings: their relations and continuity properties, J. Nonlinear Convex Anal., 10 (2009), 131-147. 2.1

[4] Y. Arfat, P. Kumam, P. Sa Ngiamsunthorn, M. A. A. Khan, H. Sarwar, H. Fukhar-ud-Din, Approximation results for split equilibrium problems and fixed point problems of nonexpansive semigroup in Hilbert spaces, Adv. Difference Equ., 2020 (2020), 21 pages. 1, 1

[5] A. Beck, M. Teboulle, A fast iterative shrinkage-thresholding algorithm for linear inverse problems, SIAM J. Imaging Sci., 2 (2009), 183-202. 1

[6] E. Blum, W. Oettli, From optimization and variational inequalities to equilibrium problems, Math. Student, 63 (1994), 123-145. 1, 2.6, 2.8

[7] F. E. Browder, W. V. Petryshyn, Construction of fixed points of nonlinear mappings in Hilbert space, J. Math. Anal. Appl., 20 (1967), 197-228. 1

[8] C. Byrne, Iterative oblique projection onto convex subsets and the split feasibility problems, Inverse Problem, 18 (2002), 441-453. 1

[9] L.-C. Ceng, J.-C. Yao, A hybrid iterative scheme for mixed equilibrium problems and fixed point problems, J. Comput. Appl. Math., 214 (2008), 186-201. 1, 1

[10] Y. Censor, T. Bortfeld, B. Martin, A. Trofimov, A unified approach for inversion problems in intensity modulated radiation therapy, Phys. Med. Biol., 51 (2006), 2353-2365. 1

[11] Y. Censor, T. Elfving, A multi-projection algorithm using Bregman projections in a product space, Numer. Algorithms, 8 (1994), 221-239. 1

[12] Y. Censor, T. Elfving, N. Kopf, T. Bortfeld, The multi-set split feasibility problem and its applications, Inverse Problem, 21 (2005), 2071-2084 1

[13] Y. Censor, A. Motov, A. Segal, Pertured projections and subgradient projections for the multiple-sets split feasibility problem, J. Math. Anal. Appl., 327 (2007), 1244-1256. 1

[14] S. S. Chang, H. W. J. Lee, C. K. Chan, L. Wang, L. J. Qin, Split feasibility problem for Quasi-nonexpansive multi-valued mappings and total asymptotically strict pseudo contractive mappings, Appl. Math. Comput., 219 (2013), 10416-10424. $1,2.4,3.2$

[15] C. Chidume, Geometric properties of Banach spaces and nonlinear iterations, Springer Verlag Series: Lecture Notes in Mathematics, (2009). 2, 2.3

[16] P. L. Combettes, The convex feasibility problem in image recovery, Adv. Imaging Electron Phys., 95 (1996), 155-270. 1

[17] P. L. Combettes, S. A. Hirstoaga, Equilibrium programming in Hilbert spaces, J. Nonlinear Convex Anal., 6 (2005), 117-136. 1, 2.6, 2.8

[18] R. Dewangan, B. S. Thakur, M. Postolache, A hybrid iteration for asymptotically strictly pseudocontractive mappings, J. Inequal. Appl., 2014 (2014), 11 pages. 3.2, 4.1, 4.2, 4.6

[19] J. N. Ezeora, Iterative Solution of Fixed Points Problem, System of Generalized Mixed Equilibrium Problems and Variational Inclusion Problems, Thai J. Math., 12 (2014), 223-244. 1

[20] J. N. Ezeora, R. C. Ogbonna, Split feasibilty problem for countable family of multi-valued nonlinear mappings, Mathematicki Vesnik, 70 (2018), 233-242. 1, 3.2

[21] D. V. Hieu, An inertial-like proximal algorithm for equilibrium problems, Math. Methods Oper. Res., 88 (2018), $399-415$. 1

[22] C. Izuchukwu, F. O. Isiogugu, C. C. Okeke, A new viscosity-type iteration for a finite family of split variational inclusion and fixed point problems between Hilbert and Banach spaces, J. Inequal. Appl., 2019 (2019), 33 pages. 2.1

[23] C. Izuchukwu, C. C. Okeke, F. O. Isiogugu, A viscosity iterative technique for split variational inclusion and fixed point problems between a Hilbert space and a Banach space, J. Fixed Point Theory Appl., 20 (2018), 25 pages. 1

[24] Q. Liu, Convergence theorems of the sequence of iterates for asymptotically demicontractive and hemicontractive mappings, Nonlinear Anal., 26 (1996), 1835-1842. 3.2, 4.1

[25] L. V. Long, D. V. Thong, V. T. Dung, New algorithms for the split variational inclusion problems and application to split feasibility problems, Optimization, 68 (2019), 2335-2363. 1

[26] Z. Ma, L. Wang, An algorithm with strong convergence for the split common fixed point problem of total asymptotically strict pseudocontraction mappings, J. Inequal. Appl., 2015 (2015), 13 pages. 1

[27] E. Masad, S. Reich, A note on the multiple-set split feasibility problem in Hilbert spaces, J. Nonlinear Convex Anal., 8 (2007), 367-371. 1 
[28] S. Plubtieng, K. Sombut, Weak convergence theorems for a system of mixed equilibrium problems and nonspreading mappings in a Hilbert space, J. Inequal. Appl., 2010 (2010), 12 pages. 1

[29] B. T. Polyak, Some methods of speeding up the convergence of iteration methods, Ussr Comput. Math. Math. Phys., 4 (1964), 1-17. 1

[30] Y. Shehu, Fixed Point Solutions of Generalized Equilibrium Problems for Nonexpansive Mappings, J. Comput. Appl. Math., 234 (2010), 892-898. 1

[31] K. Sitthithakerngkiet, J. Deepho, J. Martínez-Moreno, P. Kumam, Convergence analysis of a general iterative algorithm for finding a common solution of split variational inclusion and optimization problems, Numer. Algorithms, 79 (2018), 801-824. 1

[32] S. Takahashi, W. Takahashi, Strong convergence theorem for a generalized equilibrium problem and a nonexpansive mapping in a Hilbert space, Nonlinear Anal., 69 (2008), 1025-1033. 1

[33] C. Udrişte, Convex Functions and Optimization Methods on Riemannian Manifolds, Kluwer Academic Publishers Group, Dordrecht, (1994). 1

[34] H.-K. Xu, Iterative methods for split feasibility problem in infinite dimensional Hilbert spaces, Inverse Problems, 26 (2010), 17 pages. 1

[35] L. Yang, S.-S. Chang, Y. J. Cho, J. K. Kim, Multiple-set split feasibility problems for total asymp-totically strict pseudocontractions mappings, Fixed Point Theory Appl., 2011 (2011), 1-11. 1, 2.1, 3.2

[36] J. Zhao, D. Hou, A self-adaptive iterative algorithm for the split common fixed point problems, Numer. Algorithms, 82 (2019), 1047-1063. 1 